\section{REFERENCES}

Blackburn, C. R. B. (1953). Australasian Annals of Medicine, 2, 78.

Blendis, L. M., Williams, R., and Kreel, L (1969). Gut, 10, 433.

Cochrane, A. L., and Garland, L. H. (1952). Lancet, 2, 505 .

Dell, J. M., jun., and Klinefelter, H. F. (1946). American fournal of Medical Sciences, $211,437$.

Fletcher, C. M. (1952). Proceedings of the Royal Society of Medicine, 45, 577 .

Godfrey, S., Edwards, R. H. T., Campbell, E. J. M., Armitage, P., and Oppenheimer, E. A. (1969). Thorax, 24, 4

Kreel, L., and Mindel, S. (1969). British fournal of Radiology, 42, 830. McCready, V. R., and Ahuja, S. (1969). Proceedings of the Royal Society of Medicine, 62, 794.
McCready, V. R., Gwyther, M. M., and Stringer, A. (1969). In Medical Radioisotope Scintigraphy, vol. 2, p. 419. Vienna, International Atomic Energy Agency.

Riemenschneider, P. A., and Whalen, J. P. (1965). American fournal of Roentgenology, Radium Therapy and Nuclear Medicine, 94, 462.

Rosenbaum, H. D., Lieber, A., Hanson, D. J., and Pellegrino, E. D. (1964). American fournal of Roentgenology, Radium Therapy and Nuclear Medicine, 91, 903.

Smyllie, H. C., Blendis, L. M., and Armitage, P. (1965). Lancet, 2,

Whalen, J. P., Berne, A. S., and Riemenschneider, P. A. (1969), Radio$\log y, 92.466$.

Wyman, A. C. (1954). American fournal of Roentgenology, Radium Therapy and Nuclear Medicine, 72, 51

\title{
Erythropoietic Protoporphyria:* IV. Protection from Sunlight
}

\author{
RAMON M. FUSARO, $†$ M.D., PH.D. ; WALTER J. RUNGE, $\ddagger$ M.D., PH.D.
}

Summary: Dihydroxyacetone and lawsone, in a vanish$N$ ing cream base, applied to the skin was found to protect patients with erythropoietic protoporphyria against sunlight. The use of the same ingredients in a $50 \%$ isopropyl alcohol/water solution did not induce adequate light protection. The chemically induced ultraviolet light filter in the stratum corneum allowed these patients to change their lives from an "indoor" to an "outdoor" environment.

\section{Introduction}

Several years ago we introduced a new concept of protection against ultraviolet light (Runge and Fusaro, 1962; Fusaro and Runge, 1962). That concept consists of chemically altering the stratum corneum so that the keratin has new ultraviolet transmission characteristics. The new keratin is produced by chemically reacting a dihydroxyacetone/naphthoquinone mixture with the skin at least 24 hours before exposure to sunlight. This altered keratin reduces significantly the quanta of ultraviolet light penetrating the skin, thereby redusing the possibility of a photodynamic effect of ultraviolet light on the porphyrin molecule in the skin. We here deal with the application of this concept for the protection of erythropoietic protoporphyric patients against sunlight.

\section{Materials and Methods}

The patients were given a topical mixture of $3 \%$ dihydroxyacetone and $0.13 \%$ lawsone in a chemically compatible vanishing-cream base (Squibb Experimental Base). The cream was made by adding the dihydroxyacetone powder direct to the cream and adding the lawsone dissolved in a small quantity of isopropyl alcohol $(5 \mathrm{mk} . / \mathrm{ml}$.). The active principles were also used in a $50 \%$ solution of isopropyl alcohol and distilled water. If the solution was used it was dispensed as two solutions in order to assure stability; $6 \%$ dihydroxyacetone in $50 \%$ solution of isopropyl alcohol and distilled water and $0.25 \%$ lawsone in $50 \%$ solution of isopropyl alcohol and distilled water. The day the patient was to be treated he mixed equal parts of the two solutions and sprayed (aerosol spray) the final mixture on the skin.

\footnotetext{
* The value of normal erythrocyte protoporphyrin is $15-65 \mu \mathrm{g} . / 100 \mathrm{ml}$. of blood and not the printer's error of $565 \mu \mathrm{g}$. as seen in our first article (Peterka et al., 1965).

† Associate Professor, Department of Dermatology, University of Minnesota Medical School, Minneapolis, Minnesota, U.S.A.

¥ Assistant Professor, Department of Pathology, University of Minnesota Medical School, Minneapolis, Minnesota, U.S.A.
}

The patients used the topical therapy after their cutaneous eruption had cleared. Any eruption before the protective treatment was given was treated with topical steroid therapy and by avoiding sufficient light exposure to cause symptoms. Steroid therapy was discontinued before the protective regimen was started. This precaution of clearing the eruption was necessary as an effective ultraviolet light filter can be induced only into an intact stratum corneum. The topical preparations were applied six to eight times a day for the first 48 hours. Thereafter each patient applied the preparations three times a day for the next five days. After the first week he was allowed to go out in the sunlight for what he considered "a prolonged period of time." In his past experience sunlight exposure for this period of time was usually followed by an outbreak of cutaneous symptoms or eruption. After the first exposure the patient applied the topical therapy one to four times a day, depending on his degree of light sensitivity. If the protection was judged inadequate because of excessive light exposure the therapeutic regimen was reinstituted; but in addition the number of topical applications was increased.

The patient was instructed not to wash his skin within one hour after the application. In some patients all the applications were applied late in the day (after 18.00 hours) with at least one hour between the applications. When the patient bathed he used only soap to clean his skin and not a "washcloth" or "scrub-brush." When drying his skin after a bath, he was to "pat his skin dry" and not "vigorously rub or towel down."

In attempting to set up criteria for judging degree of protection by the amount of sunlight the patient could tolerate it was apparent that the measure of time should be simple, be realistic, and reflect his clinical experience. An endpoint, determined by the patient, of the minimal amount of sun exposure needed to produce symptoms seemed to be the simplest measure of the efficiency of protection. The patient invariably remembered that experience in which he had the least protection. That clinical exposure usually represented the most drastic exposure to sunlight. An estimation of an average of the minimum did not appreciably clarify the situation as the following variables had to be considered in determining the average: (1) day of the year; (2) time of day in which exposure was made; (3) the condition of the surrounding environment-that is, buildings, trees, etc.; (4) weather conditionsthat is, type and percentage of cloud cover; (5) the amount of clothes-that is, hat, long or short sleeves, type of material-and the length of time each item was worn during the exposure -for example, was the hat worn all the time?; and (6) each patient's disease had a different degree of photosensitivity, depending on the severity of the chemical abnormality. It was apparent that an integration of these variables into a mean- 
ingful equation would be complex and contribute little when the difference in the time needed to produce symptoms in the unprotected and protected states must be great or the patients would not use the sunscreen method.

The case numbers used in this report are the same as in the first report (Peterka, Fusaro, Runge, Jaffe, and Watson, 1965).

\section{Results}

The Table summarizes the amount of protection received by each patient. One (Case 1) has been using the therapy for six years. Previously all other topical sunscreens failed to give protection. In addition they subjectively were judged to be either cosmetically or physically objectionable. After protection with the present preparation all patients were able to change their daily lives from one of predominantly "indoors" to that of "outdoors." The children (Cases 1, 4, 5, 6, and 7) were able to go swimming and enjoy other outdoor sports for several hours a day for the first time in their lives.

Protection from Dihydroxyacetone/Lawsone Treatment

\begin{tabular}{|c|c|c|c|c|}
\hline \multirow{2}{*}{$\begin{array}{l}\text { Case } \\
\text { No. }\end{array}$} & \multirow{2}{*}{$\begin{array}{l}\text { Topical } \\
\text { Vehicle }\end{array}$} & \multirow{2}{*}{$\begin{array}{l}\text { No. of Applications } \\
\text { per Day }\end{array}$} & \multicolumn{2}{|c|}{$\begin{array}{l}\text { Time Necessary to Produce Symptoms } \\
\text { or Lesions* }\end{array}$} \\
\hline & & & $\begin{array}{l}\text { Before Protection } \\
\text { (Hours) }\end{array}$ & $\begin{array}{l}\text { After Protection } \\
(\text { Hours } \dagger)\end{array}$ \\
\hline $\begin{array}{l}1 \\
2 \\
3 \\
4 \\
5 \\
6 \\
7\end{array}$ & $\begin{array}{l}\text { Cream } \\
\text { Solution } \\
\text { Cream } \\
\text { Solution } \\
\text { Cream } \\
\text { Cream } \\
\text { Cream } \\
\text { Cream } \\
\text { Cream }\end{array}$ & $\begin{array}{c}1-2 \\
2-3 \\
1 \\
3 \\
2 \\
1 \\
4 \\
4 \\
1-4\end{array}$ & $\left\{\begin{array}{c}1 / 4 \\
<1 / 6 \\
1 / 3 \\
1 / 3 \ddagger \\
1 \\
1 \\
2\end{array}\right.$ & $\begin{array}{c}>5 \\
1 / 2 \\
>3 \\
<1 / 4 \\
>5 \\
>4 \\
8 \\
59 \\
>8\end{array}$ \\
\hline
\end{tabular}

*Time between 8 a.m. and 6 p.m. at a latitude of $45^{\circ}$ north during spring, summer and early fall.

This is minimal hours of protection.

before using protection: however, he could tolerate only 20 minutes.
§If this patient was out in sunlight during the middle of the day for six hours he developed a faintly visible erythematous eruption.

The apparent differences (see Table) in the protection afforded by the use of the two therapeutic vehicles suggest that in these patients the alcohol/water vehicle evaporates so rapidly on the cutaneous surface that there is probably not sufficient time for a complete chemical reaction to take place between the active ingredients and the stratum corneum. The cream vehicle probably acts as a barrier to prolong the available time for the reaction to occur. Cases 1 and 2 show the differences in protection resulting from use of the two vehicles. Before using the solution Case 1 used dihydroxyacetone/ lawsone in the cream base and received from 5 to 10 hours of light protection a day depending on the amount of ambient sunlight, the time of day, the type of clothes worn, and adherence to the application schedule. On the alcohol/water vehicle virtually no protection was given. The patient was restarted on dihydroxyacetone/lawsone in the cream base and acquired as much protection as previously attained.

\section{Discussion}

Donaldson, Donaldson, and Rimington (1967) reported that there was no topical sunscreen agent which absorbed effectively in the area of $400 \mathrm{~m} \mu$ for the protection of patients with erythropoietic protoporphyria. The ultraviolet light transmission data of stratum corneum treated with dihydroxyacetone/ juglone show a significant reduction of $400 \mathrm{~m} \mu$ through the treated keratin (Runge and Fusaro, 1962; Fusaro, Runge, Lynch, and Watson, 1966). Our clinical observations demonstrated and verified that the new keratin filter appreciably reduces the transmission of electromagnetic radiation through the stratum corneum of our protoporphyric patients.
Donaldson et al. (1967) stated that two of their patients felt that they received some protection from "the application of dihydroxyacetone/lawsone lotion to exposed areas." The authors, however, discounted their patients' remarks. The conflicting experience of these authors might be explained by several factors. The most obvious reason is that they shared our initial experience-that is, the solution failed to induce sufficient protection into the skin for the reasons cited above. In addition, it is not known how they made or stabilized their "dihydroxyacetone/lawsone lotion" to prevent chemical deterioration. As previously reported (Fusaro et al., 1966), the two active ingredients will react with one another, especially in the presence of heat and excessive water; therefore the "storage life" of the final mixture is very precarious.

The method of application of the sunscreen was not mentioned by Donaldson et al. (1967). Patients have been indoctrinated for years with the concept that any sunlight-protective agent is placed on the skin before going out into the sunlight as the agent absorbs ultraviolet light. The dihydroxyacetone/lawsone method of protection is different. It involves a new principle and requires re-education of the patients, as the active ingredients chemically induce an ultraviolet light filter into the stratum corneum by a chemical reaction with the keratin. Therefore our patients used the medication late in the day before bedtime and applied it for about a week before exposure to the sun for any prolonged period. The advantage of this therapy is that the medication remains at home and the protection does not have to be applied every time one goes out into the sunlight or reapplied several times a day during outdoor activities. The induced protection in the stratum corneum cannot be washed off by soap and water. It disappears with normal keratin shedding; therefore it can be renewed in the evening or bedtime. Another decided advantage of this induced filter is that the patient can indulge in the most strenuous outdoor activity without interference in sweating, as with a greasy ointment. In addition, since protection is built into the keratin, sweating does not rinse off the protection. The most important factors in acceptability by the patient are the lack of greasiness, non-interference in sweat-gland function, the protective substance did not rinse off while swimming, the skin had a chemical colour simulating a tan, and the applications were applied at home, not outdoors while engaged in sport activities.

As mentioned previously, the degree of sunlight protection received by our patients depended on several factors : amount of ambient sunlight, etc. In addition we live in Minneapolis, Minnesota, at $45^{\circ}$ north latitude; therefore the total amount of electromagnetic radiation available to us is much less than that in other areas of the United States (Daniels, 1967). Also, there are fewer sunny days in Minnesota than in many other areas of the United States. In fact, the sun shines only $58 \%$ of the possible sunshine hours (U.S. Weather Bureau, Minneapolis, Minnesota), so there are definite limits to the amount of protection we can induce into the skin by the regimen described above. It is obvious our patients cannot sunbathe 12 hours a day. Nevertheless, before using the dihydroxyacetone/ lawsone method, their existence was essentially "indoors." Now it is substantially "outdoors"- that is, they "may sit $i$ ' the centre, and enjoy bright day" (Milton's Comus, 1. 381).

\section{REFERENCES}

Daniels, F., jun. (1967). In Therapeutic Electricity and Ultraviolet Radiation, 2 nd ed., edited by S. Licht, p. 379. New Haven, Licht.

Donaldson, E. M., Donaldson, A. D, and Rimington, C. (1967). British Medical fournal, 1, 659.

Fusaro, R. M., and Runge, W. J. (1962). Journal of the American Medical Association, 182, 1120 .

Fusaro, R. M., Runge, W. J., Lynch, F. W., and Watson, C. J. (1966). Archives of Dermatology, 93, 106.

Peterka, E. S., Fusaro, R. M., Runge, W. J., Jaffe, M. O., and Watson, C. J. (1965). Fournal of the American Medical Association, 193, 1036. Runge, W. J., and Fusaro, R. M. (1962). fournal of Investigative Dermatology, 39, 431 . 